\title{
Medical System for X-Ray Analysis
}

\author{
Bárbara Emma Sánchez ${ }^{1}$, Alberto Jaramillo² ${ }^{2}$ Guillermo Vara ${ }^{1}$ \\ ${ }^{1}$ Benemérita Universidad Autónoma de Puebla, Facultad de Computación, Puebla, México \\ ${ }^{2}$ INAOE, Luis Enrique Erro Tonanzintla, Puebla, México \\ brinza@hotmail.com
}

\begin{abstract}
Problems within the bone system that a patient experiences when undergoing cancer treatment are always a cause for concern, because they can trigger other serious diseases such as osteoporosis, among others, due to the loss of bone density in the system, caused by bone metastasis, that is why it is necessary to give them a detailed follow-up and apply different treatments to avoid this type of problems, for this reason the main reason for the development of a system of this kind that allows to have better efficiency at the time of medication and both the patient and the specialist doctor are more certain that the results are obtained from a system that allows to calculate the difference between two images, one taken at the beginning of the treatment and a later time, obtaining graphs And numerical percentages that the medical specialist can understand to make a better decision knowing that's on from a reliable and secure source.
\end{abstract}

Keywords. System, rotations, histograms, radiography, Python.

\section{Introduction}

The present project develops a visual medical system for the optimization of the medication process to patients with cancer problems, who have been prescribed a treatment to avoid the deterioration of the bones caused by the aforementioned disease.

Develop a medical system under the Python programming language that works as an aid to doctors in determining if a treatment is working on a patient who is undergoing certain treatment.

Has as purpose:

- Time optimization for doctors and users.

- Assist in the diagnosis of medical treatments.

- Improve the care and efficiency of diagnosis.

For the programming of the medical system in its version 1.0, the system was developed on the programming language Python which is a language that has efficient data structures, high level and a simple but effective approach in object programming which 
makes it a language suitable for the realization of the system and due to the great support with which it has within the community has an extensive number of libraries to support when processing and interpreting the images.

The medical system was developed on a computer with the following characteristics:

- Operating System: Windows 10 x64 with 8 GB Ram,

- Python: 2.7.13,

- OpenCV: 3.2,

- PyInstaller: 3.2,

- Numpy: 1.3 ,

- IDE: SublimeText 3126 for 64-bit.

Below is a brief description of the elements and libraries that were necessary to install to carry out the programming.

\section{$2 \quad$ Starting the Program}

From the wx.Python library for window management, we create the startup window where the user is presented with the system (Fig. 1) and invited to enter [1].

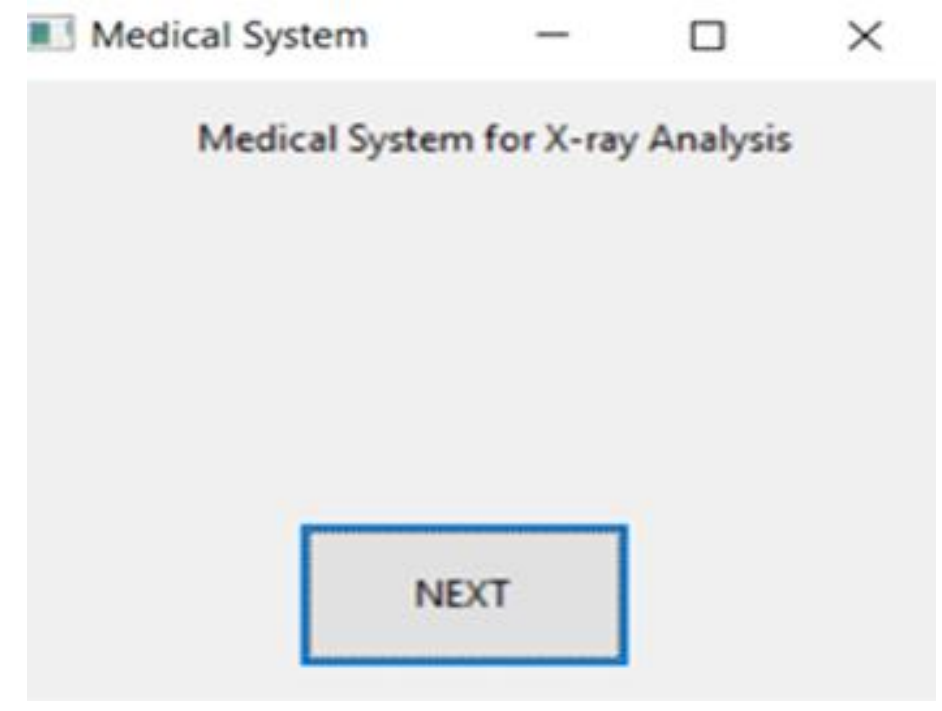

Fig. 1. Start-up interface.

Once the user has entered the system, a user interface (Fig. 2) has been created that has different options for the use of the software. In order to begin to occupy the software correctly, it is always necessary to have the images loaded otherwise the system will throw an error.

Where the buttons are created for operations from the wx.Buttons classes and are added to the interface with the "self.sizer.Add ()" method that is obtained from the main 
interface named "self" and the class "Sizer" from which the "Add" method is retrieved, which receives the widget information that will be added to the main window, as shown in figure 2.
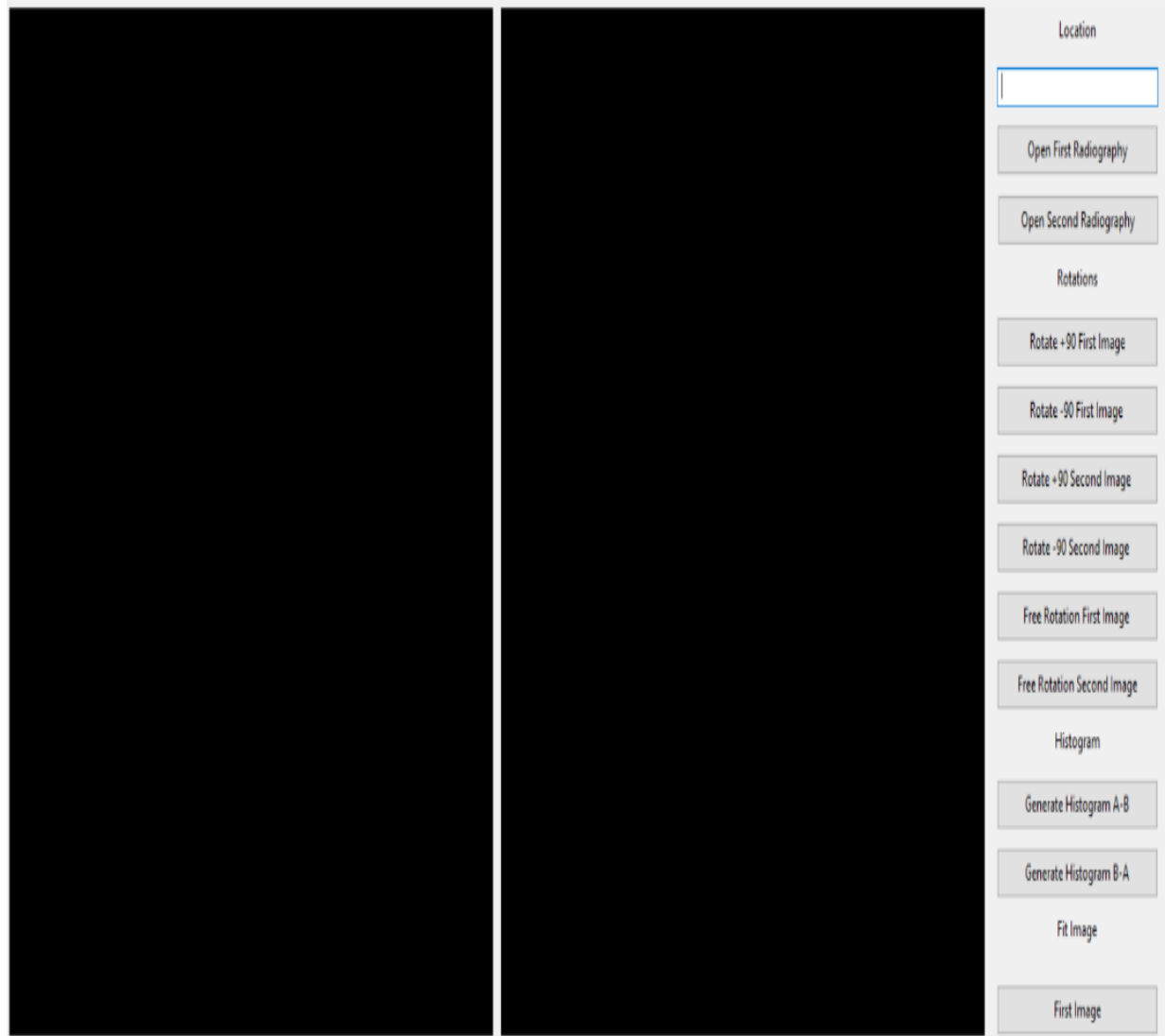

Open first Radiography

Open Second Radiogaphy

Retations

Rotite + MV Firstlmage

Rotate - N Firit Image

Rotate +NOS Scond I mage

Rotte - No Second Image

Free fortiton first mage

Fire Rototion Second I mage

Histogram

Generte Hitogram A.B

GeneruteHistogram B-A

Fitmage

firtimage

Secondmage

Fig. 2. Main interface.

Then with wx.Python we create from the left side what are the windows of 600x600 where the $\mathrm{x}$-rays chosen by the user in a $*$.jpg format are uploaded, these images can be loaded with the buttons that are on the right side where they are Indicates to which image box is destined, to be able to load the image the OpenCV library was used to be able to pass the image of a format * .jpg to a format BitMap with which you can carry out operations later, Image to the size of the aforementioned box, in order to make this adjustment and not lose the proportions of the image.

This allows the user to present an image adapted to $600 \times 600$ without losing the appearance of the image (Fig. 3). 


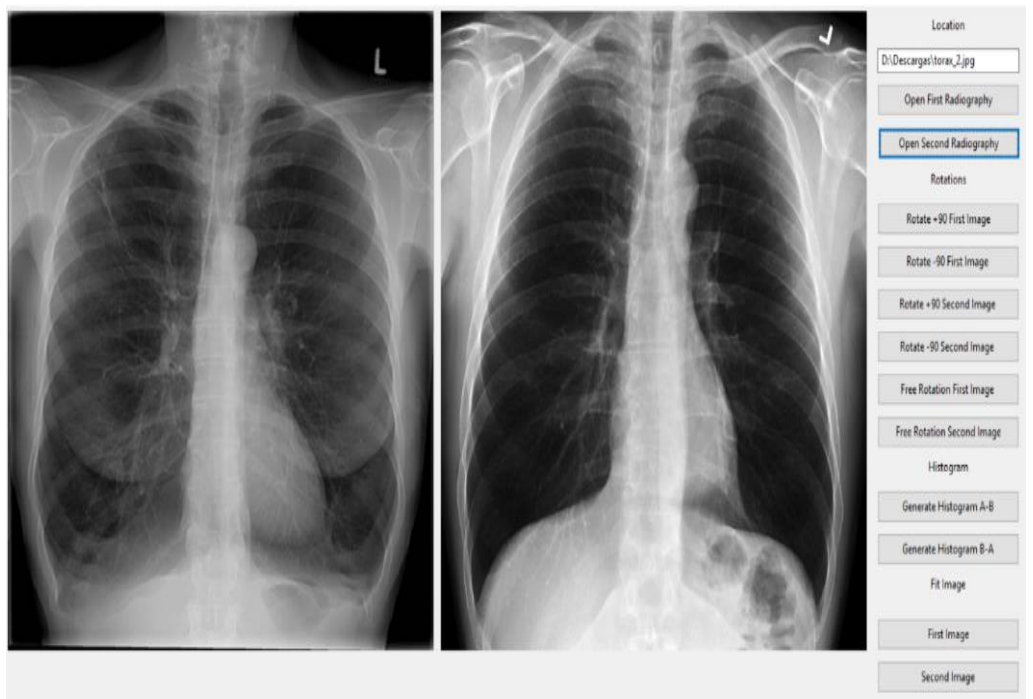

Fig. 3. Presentation of the X-ray.

\section{Operations on the Images}

\subsection{Rotations}

Within the system interface and for greater convenience when working with x-rays, the system allows rotations of $90^{\circ},-90^{\circ}$ and $0-360^{\circ}$ (Fig. 4), so that at the moment to load two images and need to adjust the position of some of them for their correct analysis can be performed without having to occupy any external tool. [2].

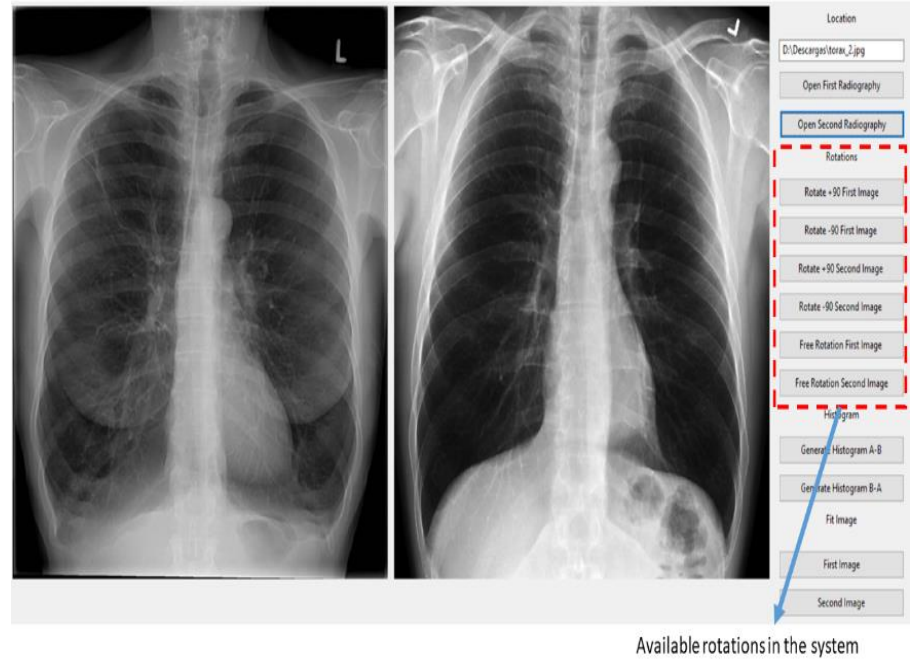

Fig. 4. Rotations available. 
To rotate 90 and $-90^{\circ}$ to use these functions, it is only necessary to press one of the buttons "Rotate 90" (Fig. 5) depending on the case and the image to be rotated, it must be taken into account that once. That the rotation operation is performed on the image is over writes the original image for use in the next operation, so it is advisable to make a backup of your original file before performing any operation with the image.

The implementation of the aforementioned rotations, were performed through OpenCV by means of the function "getRotationMatrix2D (center, angle, scale)", which asks us for two points corresponding to the center of the image which were calculated and saved in a tuple by calculating them from the division of the width of the image between two for the first point and the height of the image for the second point.

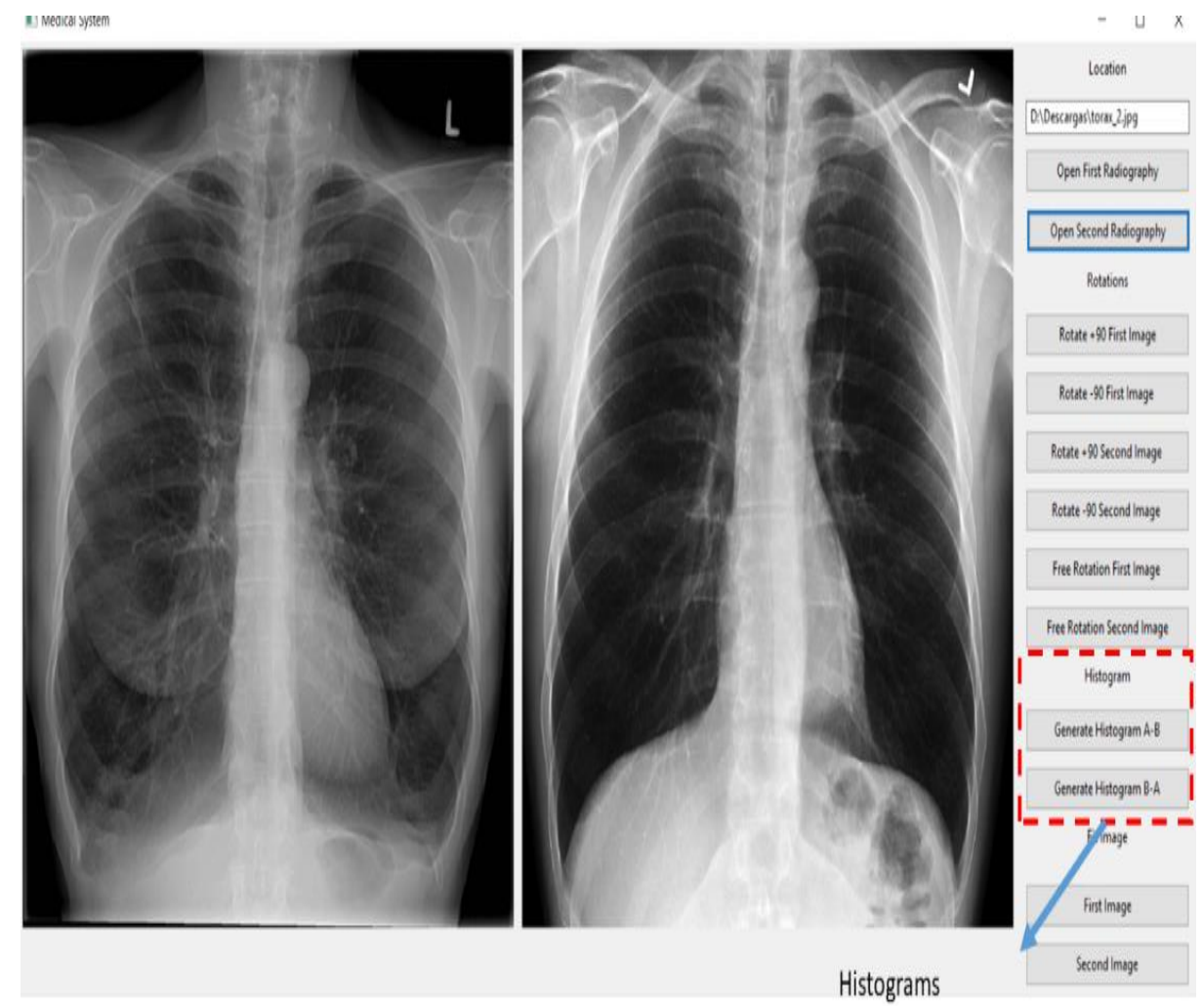

Fig. 5. Default Rotations

To use the free rotation functions, it is only necessary to press one of the buttons "Free rotation" (Fig 6) depending on the case and the image to be rotated, once the button is pressed the system launches (Fig. 7) where it is necessary to indicate by degrees the rotation in which the degrees of rotation go from $0^{\circ}$ to $360^{\circ}$ taking into account that all rotation goes in the direction of the clock [3] . 


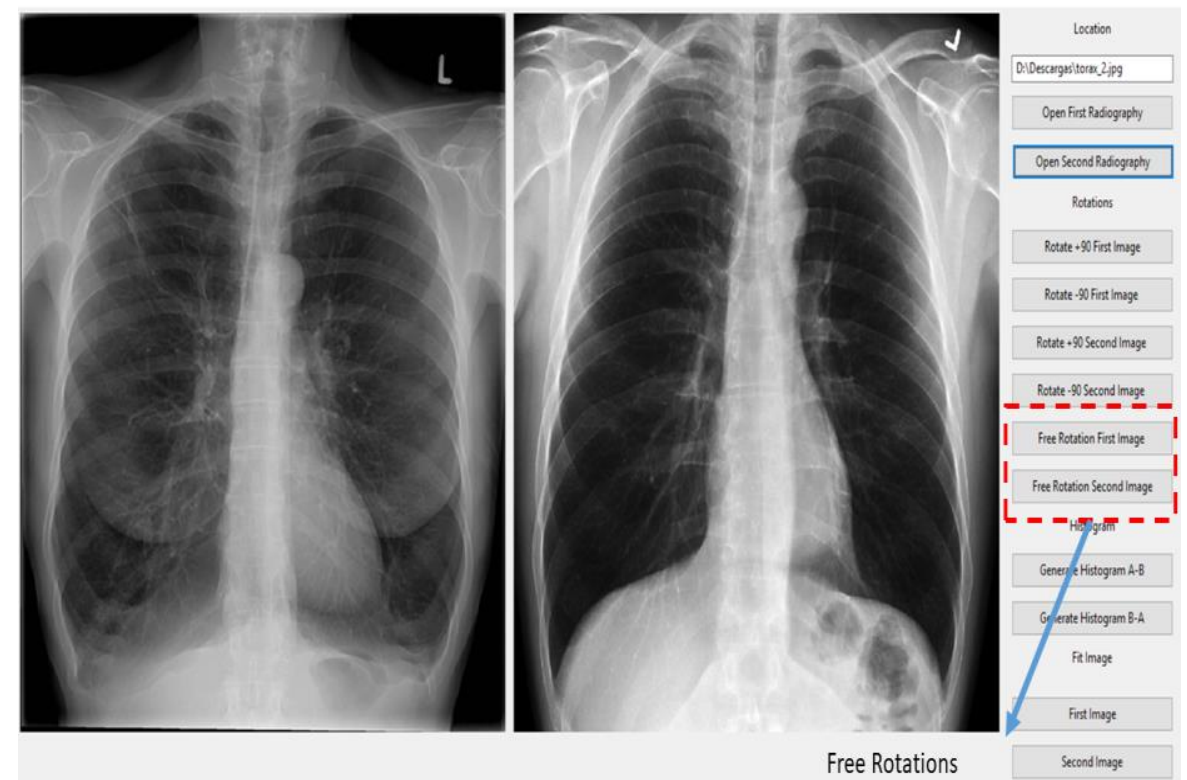

Fig. 6. Free rotation.

It is necessary to take into account that once the operation of rotation on the image is performed, the image is written to originate for using in the next operation, therefore it is advisable to make a backup of its original file before performing any operation with the image.
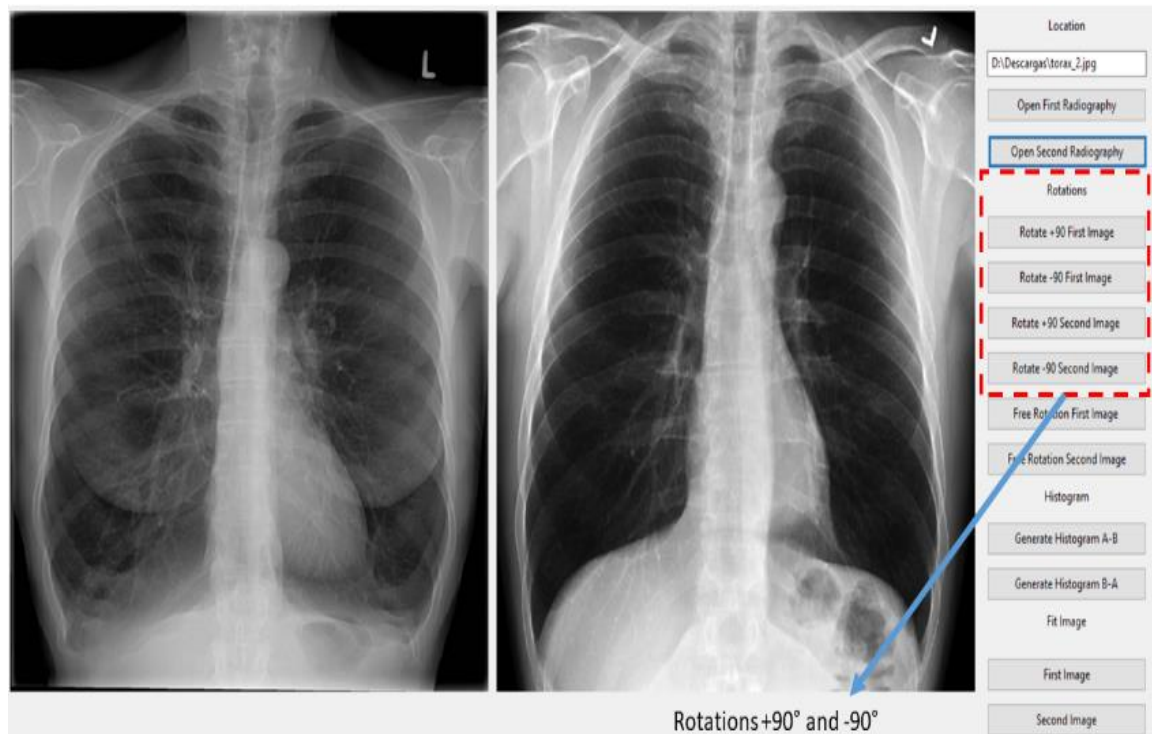

Fig. 7. Rotational angles. 
For the implementation of the free rotation, at the time of launching the window to indicate the degrees of rotation, a new window was created with wx.Python which receives the values for the rotation and once the user clicks "Accept "The system makes a step of parameters towards the corresponding method which receives them and passes them to the function of OpenCV that by means of the function of" getRotationMatrix2D (center, angle, scale) ", performs the rotation.

\section{Adjustment on the Imagen}

The use of this tool was developed with the aim of allowing users to trim the image in a given case that the image has parts that will not be relevant when performing our analysis or in case you only want to analyze certain part of the image and discard some other. To use this tool, we have to go to the "Image Adjustment" section (Fig. 8) and select the image that we want to adjust for which we skip a window (Fig. 9) with the image to be worked once we have that window, we proceed to press the beginning of where we want to cut and drag without releasing the mouse towards the other end that we want to have of our image $[1,2]$.

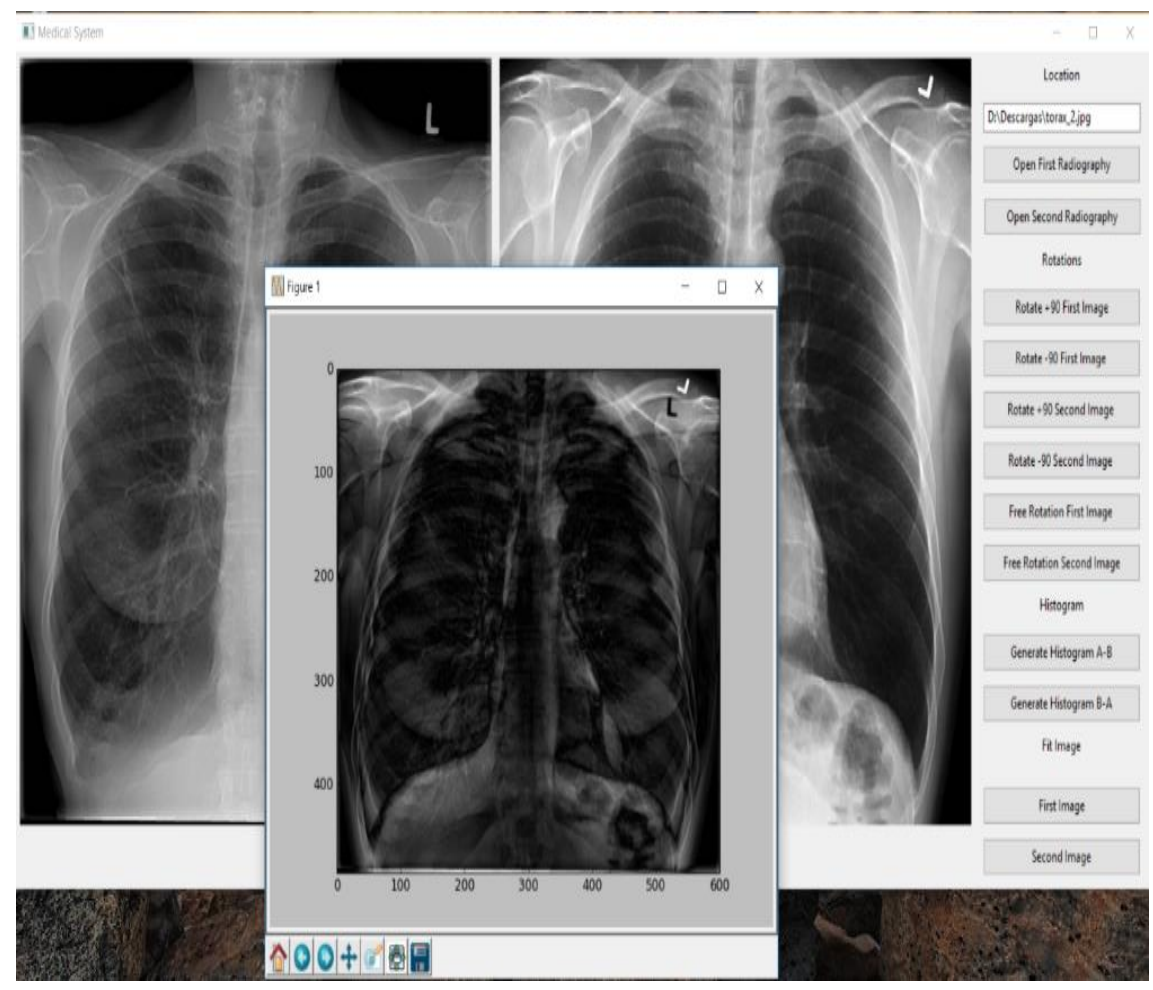

Fig. 8. Adjusting images. 


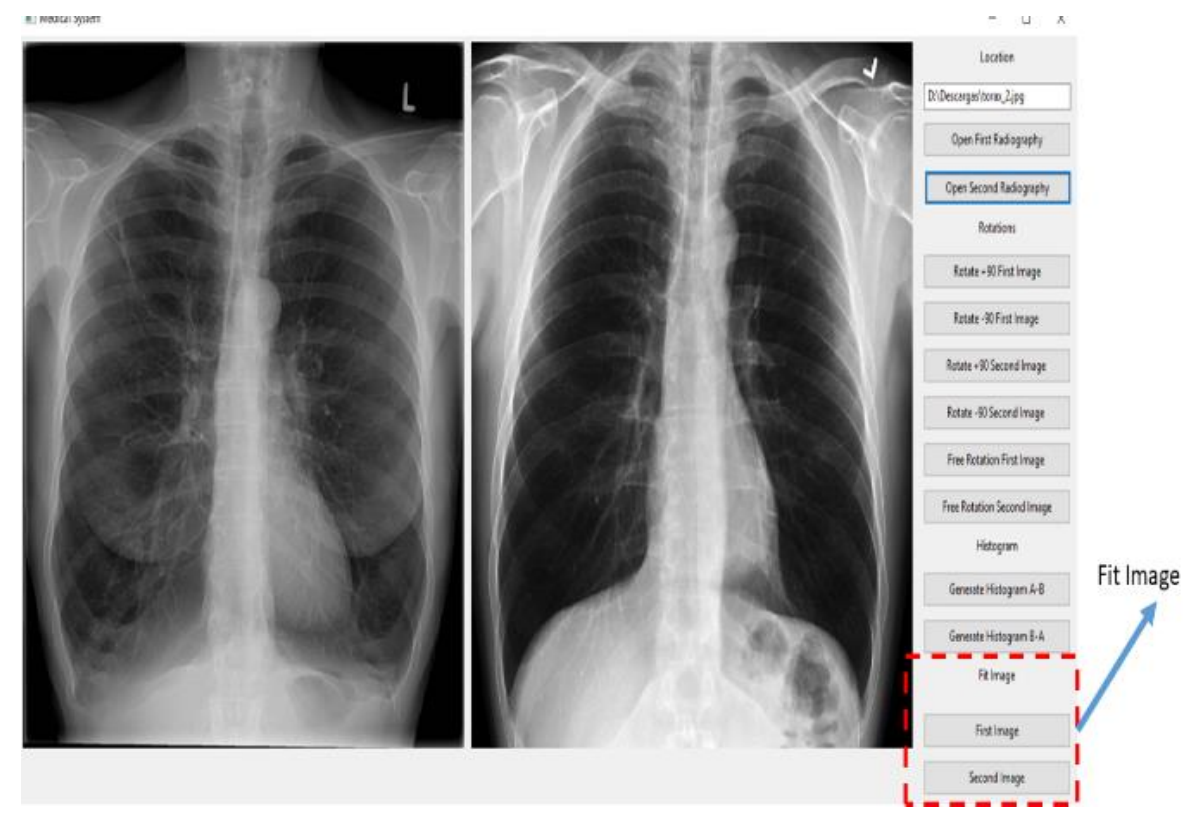

Fig. 9. Picture adjustment interface.

For the development of this section the OpenCV library was occupied, first what was done was to initialize a window in which the user through a pointer begins to select the point at which point he wants to cut. The image, once the user releases the right click of the mouse as an indication that all points were captured the system through the "setMouseCallback" method obtains the coordinates of the points and save them in an arrangement that we pass to the method " Get_clicks () "where from the previously obtained points a cut of the image of rectangular form is made and we processed the points with the method" resize () "that returns to us the image cuts that we save in a temporary image variable that then it is opened directly in the main interface of the system where from then on you can work with it.

Once we release the right mouse button the system will crop the image and send it to the main window of the system to continue working with it, taking into account that the initial image will be overwritten so it is recommended to have a copy of the main image by If at some point it is necessary to reuse.

\section{Calculate Histograms and Bone Gain}

Once you have the TWO images already worked, you can calculate the histograms depending on the needs we can calculate "AB" or "BA", to perform these operations we located in the "Histogram" section (Fig. 10) and press the operation to be calculated 

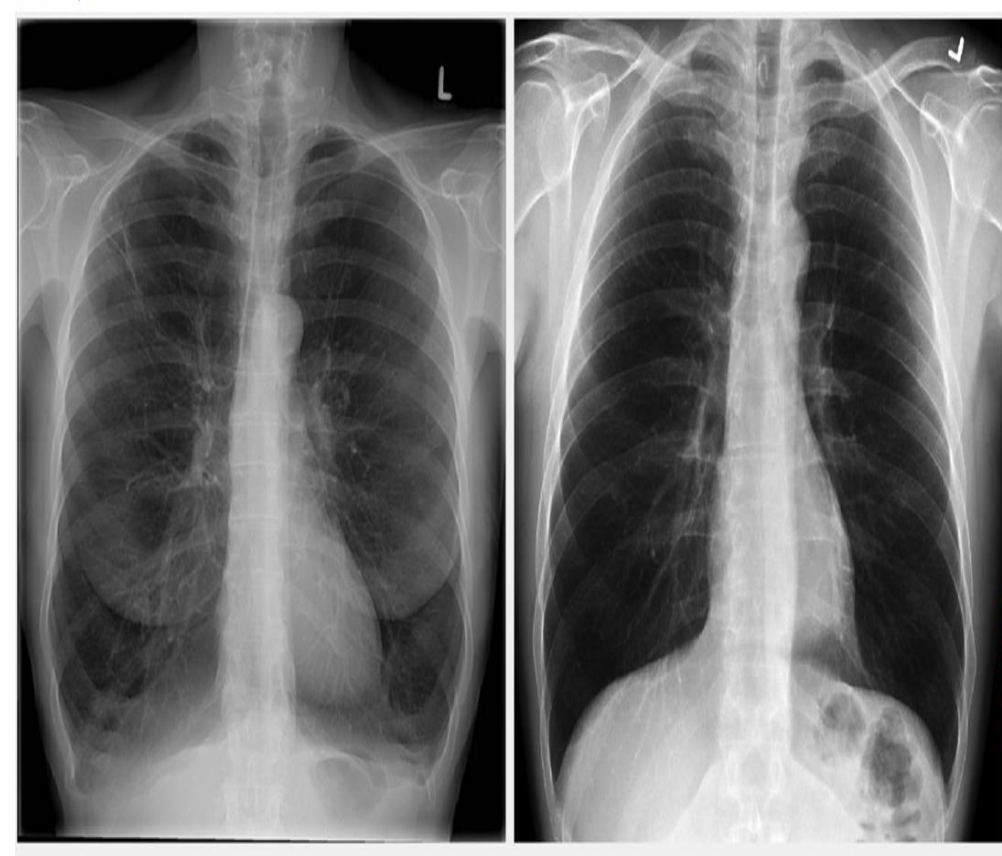

loution

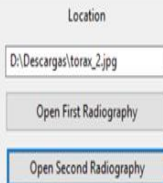

Rettions

Rotate + + OFist Imgge

Rotte- Q First Inige

Rotate +90 Second Image

Rotate -90 Second Image

Fiete Rototon first limge

Histograms

Fig. 10. Histograms.

The system calculates the histograms and the bone gain and it unfolds as follows taking into account that to calculate the histograms and the bone gain:

- Image subtraction result window (Fig. 11) in this window shows the resulting image of the subtraction and in this window you can perform operations such as "Save the image", "Configure the graphic", "Perform Zoom in a certain area "," Move the image within the graph "and" Return to its initial state ".

The implementation of the subtraction of the images was developed from the OpenCV library with the "absdiff" method, which is passed as parameters the two images that the system previously verified to be of the same size and That are in valid pixel formats. Once the subtraction of the images has been obtained, a "cv2" matrix is obtained which we convert into an image of type "wx.Image" so that it can be displayed inside the "plt" window which allows us to perform the Save, zoom and reset operations

- Results window of the bone gain (Fig. 12) this window will first show the bone gain in percentage, way followed by the average value of the gray tones of the resulting image and finally the average value of the gray tones of the initial image, taking as the initial image in case of "AB" the image "A" and in the case of "BA" the image "B". To calculate the bone gain, the matrix "cv2" is 
traversed in the rows and columns to obtain the average gray scale for each one and to be able to apply the formula:

Bone gain $=\begin{aligned} & \text { average value of the gray tones of the RTE image } \\ & \text { average value of the gray tones of the inicial image }\end{aligned} * 100$.

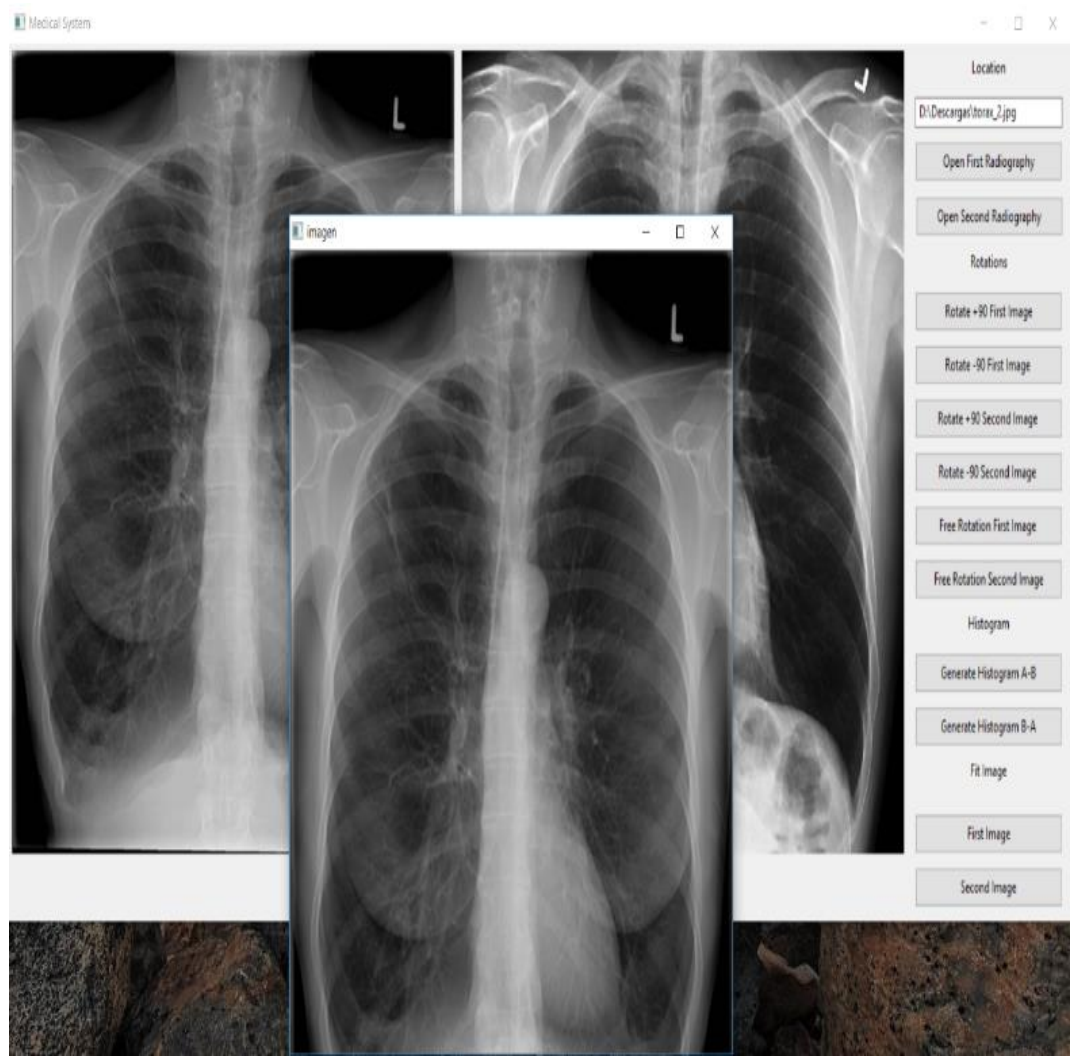

Fig. 11. Image substraction.

Histogram window (Fig. 13) this window will show the calculated histogram of the resulting image where the number of pixels is represented on the "Y" axis and the pixel's gray hue as the " $\mathrm{X}$ " axis. Within this window you can perform operations such as "Save the image", "Configure the graph", "Zoom into a specific area", "Move the image within the graph" and "Return to its initial state".

The histogram is calculated with OpenCV and the "calcHist" method, which is passed the image resulting from the subtraction and the range of values of the colors of the image, taking the case of the $\mathrm{x}$-rays that are worked on scale gray is passed a range of 0-256 in grayscale. 
Medical System for X-Ray Analysis

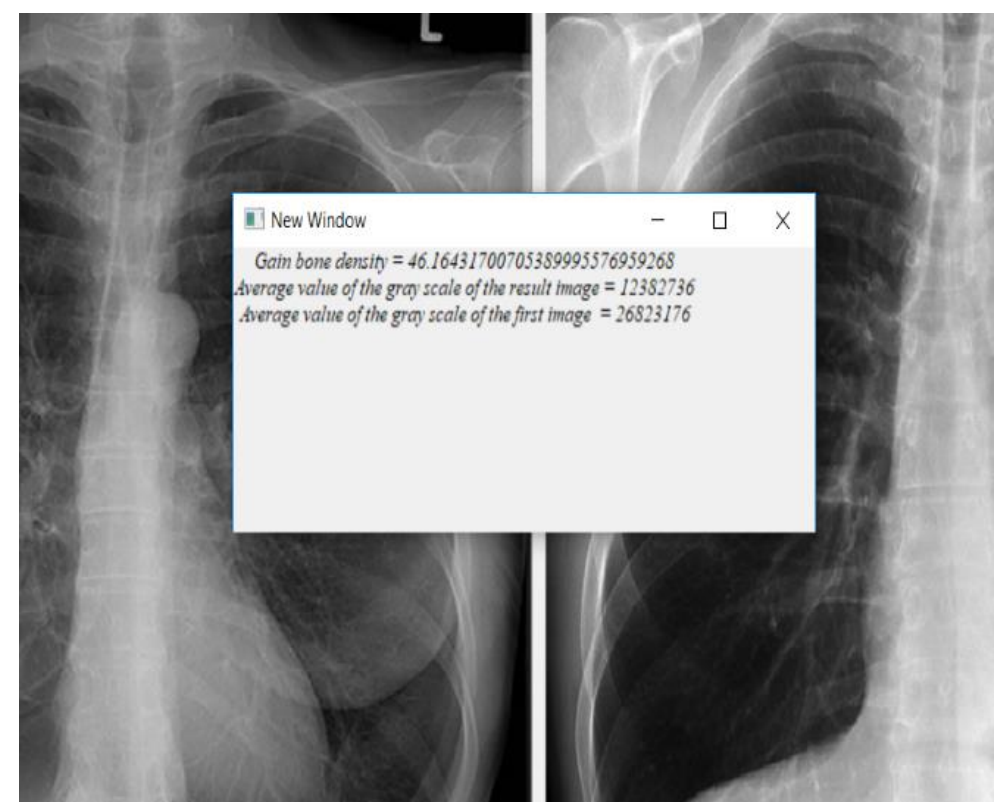

Fig. 12. Bone gain.

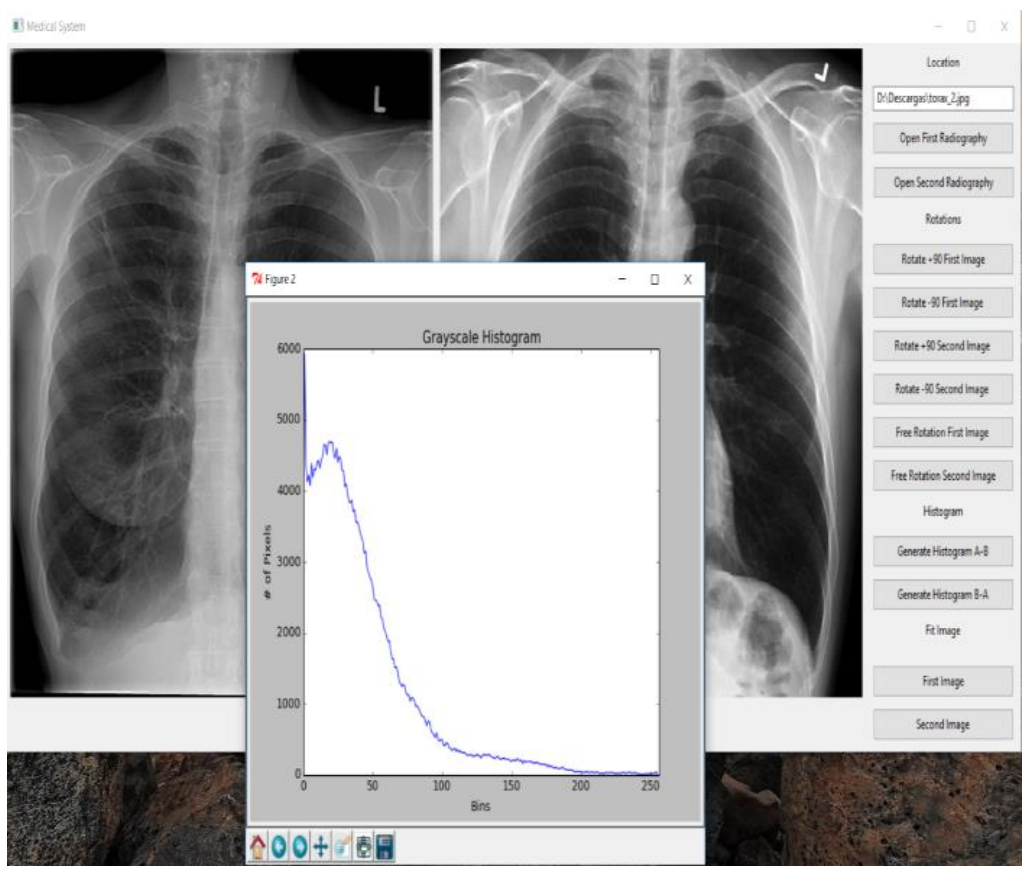

Fig. 13. Resulting image histogram. 


\section{Conclusions}

So far the results obtained with the software have been satisfactory obtaining graphs and percentages that the doctors have been able to interpret, thus fulfilling the objective of the work, which is the optimization of time for both specialist doctors and patients. And making the method of drug delivery more efficient.

According to the results obtained, it is concluded that images taken by radiography can be processed by a computer to obtain more efficient numerical values that help the decision making by the doctors.

\section{References}

1 Reiser, M., Vahlensieck, M.: Resonancia Magnética Musculoesquelética. España: Médica Panamericana (2010)

2 Bohem, B.: Spiral Model of Software Development and Enhancement. IEEE Computer, May (1988)

3 https://www.blog.pythonlibrary.org/about/ 